\title{
The Impact of Extreme Space Weather on The Rise of Biomolecules: Early Earth and exo-Earth environments
}

White paper submitted in response to the Decadal Survey on Planetary Science and Astrobiology

Primary Author: V. S. Airapetian (GSFC/SEEC and American University, DC, email: vladimir.airapetian@nasa.gov

Co-authors: G. Gronoff, SSAI/LaRC, guillaume.gronoff@gmail.com, K. Kobayashi, Yokohama National University, kobayashi-kensei-wv@ynu.ac.jp, D. Nna Mvondo, UMBC, delphine.nnamvondo@nasa.gov A. Zestos, American University, zestos@american.edu, James Kasting,Penn State, jfk4@psu.edu, Benjamin Hayworth, Penn State, bph8@psu.edu, A. Glocer, NASA/GSFC, B. Lynch, UC Berkeley, blynch@ssl.berkeley.edu, Y. Notsu, University of Colorado Boulder, Yuta.Notsu@colorado.edu, H. Maehara,NAOJ, hiroyuki.maehara@nao.ac.jp, Y.Yamashiki, Kyoto University, yosuke_y@me.com, K., G. Li, gangli.uah@gmail.com, University of Alabama at Hunstville,, G. P. Zank, University of Alabama at Huntsville, garyp.zank@gmail.com,W.C.Danchi,NASA/GSFC/SEEC, william.c.danchi@nasa.gov, K. France, University of Colorado, Boulder, kevin.france@lasp.colorado.edu, R. Navarro-Gonzalez, Universidad Nacional Autonoma de Mexico, navarro@nucleares.unam.mx, K. Herbst, Christian-Albrechts-Universität zu Kiel, herbst@physik.uni-kiel.de, M. Jin, SETI Institute, jinmeng@lmsal.com.W.Klang, NASA/GSFC, weijia.kuang-1@nasa.gov, Feng Tian, University of Colorado at Boulder, tianfengco@gmail.com

Co-signers: $Y$. Lee (UMBC)

\footnotetext{
Abstract

Local (planetary) and global-scale (heliospheric) environments fostered the conditions for prebiotic chemistry and later for biopolymers on early Earth. This white paper aims to identify and describe promising key research goals to aid the field of exoplanetary habitability for the next 20 years.
} 


\section{Motivation.}

Are we alone in the Universe? Is life unique to Earth or a common phenomenon in the Solar System and the Universe? These fundamental questions are among the greatest puzzles of astrobiology. Earth's evident long-term habitability makes it a key data point for understanding the formation of habitable worlds in the Universe. Earth could be a unique outlier, or it could be a typical rocky planet. Understanding the conditions that allowed for the emergence of life on early Earth, and whether other terrestrial type planets in our Solar System possibly also supported habitable conditions early in their histories, but failed to sustain them. Thus, identifying the conditions compatible with the presence of organic polymers in the primitive Solar System and factors promoting prebiotic chemistry will help us to understand the origin of life on our planet and the habitability of other planetary systems in our Galaxy. In the last few years, there has been a growing appreciation that the atmospheric chemistry, and even retention of an atmosphere in many cases, depends critically on the high-energy radiation and particle environments around these stars [review paper 1 and references therein]. Indeed, recent studies have shown stellar activity and the extreme space weather, such as that created by the frequent flares and coronal mass ejections (CMEs) from the active stars and young Sun, may have profoundly affected the chemistry and climate and thus the rise of habitable conditions and biomolecules on the early Earth and terrestrial type exoplanets. The goal of this white paper is to identify and describe promising key research goals to aid in blending the fields of theoretical and laboratory prebiotic chemistry with exoplanetary science to develop strategies to search for life signatures in the solar neighborhood for the next 20 years. This paper complements the white paper by Lyons et al. 2020 submitted in response to the Decadal Survey on Planetary Science and Astrobiology.

\section{Space weather from the Young Sun as a Factor of Habitability}

Based on geochemical evidence, it seems likely life started on Earth sometime before $\quad 3.5 \mathrm{Ga}$, and possibly as early as $4.3 \mathrm{Ga}$, and thus the insights on the initiation of building blocks of life can be found by studying early Earth's environments. The early Solar System was a chaotic and turbulent place, likely subject to frequent giant mpacts and the violently changing energetic flux from the young Sun. During this time, the young Sun was violently interacting with planetary environments that dynamically evolved from their cores to atmospheres. One of the challenging questions that needs to be answered is "How did solar ionizing radiation from the young Sun evolve in the first 500 Myr and how important it was for prebiotic chemistry, particularly when combined with the physico-chemical evolution of planetary atmospheres under the influence of their volcanic and tectonic activity?" Specifically, we need to know how the evolving Sun influenced the atmospheric chemistry, pressure, and climate on early Earth? When the conditions on early Earth became compatible with the efficient production of precursor 
molecules of life, their synthesis and eventually started to promote the formation of biopolymers and chemical evolution of primitive life? To answer these questions, we need to understand how planets could efficiently outgas and keep atmospheres thick enough to contain sufficient greenhouse gases to maintain liquid surface water during early periods of planetary system evolution. This is important as the young Sun was magnetically active but fainter, which could explain the warm and wet climates of early Earth, Mars, and possibly Venus.

It is further believed that mildly reducing planetary atmospheres exposed to persistent energy sources helped promote the formation of prebiotic organic compounds. Estimating how common these conditions may have been in primitive planetary environments throughout the cosmos is crucial for understanding the habitability of terrestrial-type planets and the ways we search for habitable worlds, which are central questions for NASA. The interaction and co-evolution of three basic planetary subsystems: the atmosphere, the oceans, and planetary interiors from core to mantle to crust provided geochemical feedbacks on early Earth which created favorable conditions for the emergence of life even under the range of environmental conditions that occurred during the evolution of the young Sun. In the first billion years of the Solar System's turbulent history, these conditions promoted the rise of prebiotic chemistry that served as the basis for the origin and emergence of life. Understanding how these systems dynamically interacted requires interdisciplinary, holistic studies that can only be performed through the coordinated activities and cooperation of astrophysicists, heliophysicists, geophysicists, planetary scientists, and chemists. Our study requires a systemic observational, theoretical, and experimental approach. Such an interdisciplinary approach will allow the development of a common framework to identify and characterize the interplay between the planetary (interior and atmospheric) and heliospheric environments (in space between the planets and the Sun). This requires blended observational, theoretical, and experimental approaches.

\section{Goal \#1. Reconstructing the Magnetic Lives of the young suns}

Solar flares, coronal mass ejections (CMEs) and solar energetic particles (SEPs) produce disturbances in interplanetary space collectively referred to as space weather, which interacts with the Earth's upper atmosphere and causes a dramatic impact on spaceand ground-based technological systems [2]. Early Venus, Earth, and Mars, as well as exoplanets within habitable zones (HZs) around young and active cool dwarfs, are exposed to extreme ionizing radiation fluxes, thus making extreme space weather effects a crucial factor of habitability [3-5]. This factor should be addressed using proven methodologies developed by heliophysicists and solar physicists using data constrained multi-dimensional magnetohydrodynamic (MHD) models to model the forms of magnetic activity from the evolving Sun and solar-like stars. This includes the impact of the solar magnetic field and flows on the buildup and release of magnetic energy that heats the solar atmosphere 
forming its X-ray and Extreme UV (EUV) bright corona, the cradle of the solar wind, solar flares and coronal mass ejections (CMEs). These studies should leverage from coordinated multi-wavelength observations using the UV data from Hubble Space Telescope (HST) complemented by X-ray observations using XMM-Newton, NICER missions, and Transiting Exoplanet Survey Satellite (TESS), spectropolarimetric observations of young Sun's analogs and upcoming James Webb and Nancy Roman Space Telescopes. These data can be used as inputs and boundary conditions for multi-dimensional MHD numerical tools. The heliophysics models have high potential to provide the ionizing EUV coronal and flare driven radiation and mass fluxes from the solar wind and CMEs as a function of time during the first Gyr [1,6]. The modeled $C M E$ shock properties can then be used. Our understanding of the ionizing radiation fluxes on planetary hosts will be fueled by the following actions in the coming decade: 1. Detection and characterization of superflares on G-M dwarfs, their spectral signatures, energy partition in X-ray-to radio band, frequency and relations to spot sizes, rotation rates, and effective temperatures $[7,8]$. 2. Search for radio and optical stellar CMEs signatures by performing extended long-term observations at lower frequencies (< $10 \mathrm{MHz}$ ) with space or lunar radio missions. 3. Derive observational signatures of stellar energetic particles using modeling and statistical correlations between proxy events from solar physics [9]. 4. Characterization of $\mathrm{X}$-ray and EUV fluxes from G-M dwarfs using coordinated multi-observatory observations; 5. Reconstruction of magnetograms from active G-M dwarfs as a prerequisite to constrain space weather models [10]; 6. Development of the capability to conduct nearcontinuous simultaneous monitoring of thousands of nearby systems for radio emission (stellar CMEs, planetary auroral emissions) and optical emission (stellar flares).

\section{Goal \#2. Impact of Young Suns on Atmospheric Evolution of early (exo) Earths}

The long-term evolution of the exoplanetary atmospheres results from the interplay between solar radiation, particle, exogenic, and endogenic forces that are involved in modifying the planet's environment on a scale of billions of years. Research in the upcoming decade must address this major question as it affects the physico-chemical atmospheric changes on rocky planets and its effects on climate. The atmospheres of early Earth, Venus and Mars, and exo-Earths within respective HZs around G-M dwarfs, could be vulnerable to the high XUV fluxes from stellar flares, coronal mass ejections, dense and fast stellar winds $[1,11,12]$. The atmospheric pressure at a given evolutionary period is controlled by the outgassing rate, the removal of gases due to carbon precipitation, and atmospheric escape. This impact controls the atmospheric thickness and planetary climate that can significantly influence the evolution of (exo)planetary atmospheres [1]. Specifically, space weather can enhance the atmospheric escape of oxygen and nitrogen ions, the crucial factors of prebiotic chemistry, and the rise of biomolecules. One crucial question for exoplanetary habitability is: "How is extreme space weather important in specifying the rate of atmospheric escape of neutral and ion species and its effects on 
climate and habitability of early Venus, Earth, Mars, and (exo)planets with different surface gravities?"

We propose to address this topic by using a broad range of multi-dimensional multifluid physico-chemical atmospheric models emerging from the extension of sophisticated heliophysics tools that study the impact of XUV emission and solar winds on (exo)planetary atmospheres. These models should be validated for the extreme conditions in our solar system along with computational capabilities, laboratory experiments, and the development of mission concepts. This suggests that star-planet interactions should be modeled in a global planetary system environment with a systematic, integrated approach using theoretical and observational methods combining tools and methodologies of the four NASA science disciplines: astrophysics, heliophysics, Earth and planetary sciences.

We recommend modifications of existing space weather models for exoplanetary environments to calculate the magnetospheric response and associated atmospheric heating and escape rates. Global magnetospheric models coupled with a kinetic model of the ring current and inner plasma sheet and their coupling to the ionosphere, provide an appropriate magnetospheric response that can then be fed into a global ionosphere-thermosphere-mesosphere (ITM) model to provide the temperature and pressure, the resultant atmospheric chemistry and atmospheric and atmospheric escape rates [13]. Such coupled code suites have already been developed as a part of the Community Coordinated Modeling Center at NASA GSFC [14].

Providing appropriate stellar boundary conditions is an integral part of the recommended approach. Significant observational data for G-M dwarfs is available (e.g., [15, 16]), but is incomplete in age, spectral type, and X-ray and UV (XUV) emission. Our recommended program should combine advanced numerical and observational approaches. XUV spectroscopic data and direct measurements of stellar surface magnetic fields should be used as direct inputs for heliophysics-based multi-dimensional models of stellar coronae, winds, and CMEs [see for example, [17]). These results will provide high fidelity inputs to numerical models of the thermodynamic and chemical evolution of exoplanet atmospheres, leading to the knowledge of an exoplanet's habitable conditions over time. These models will specify the initial and boundary conditions for the coupled ionosphere-thermosphere models that describe the ion and neutral temperature and density of the coupled ionospherethermosphere systems $[4,18]$. These upper atmosphere models will provide a realistic assessment of hydrodynamic, ion, and neutral escape from atmospheres of exoplanets around cool dwarfs.

An intriguing aspect of the interaction between extreme space weather and atmospheres relates to the creation and evolution of abiotically-generated oxygen atmospheres [19]. These atmospheres can be produced during the extended pre-mainsequence phase predicted for the lowest mass stars. During this phase, and for the first few Gyr after the star begins fusing hydrogen, the star is very active, and flares could blow off the atmosphere. Alternatively, ionizing radiation (i.e., UV and XUV) could drive vigorous 
photochemistry and detectable auroral features (e.g.,[20,21]). These atmospheres are not present in our Solar System, but they may be common in HZs around K and M dwarfs, i.e. precisely the planets that will be discovered by TESS and remotely probed by JWST transit transmission and eclipse spectroscopy. A clear understanding of the evolution of these atmospheres in the context of ESW will be vital for the successful interpretation of JWST spectra [22].

\section{Goal \#3. Impact of the young Sun on Atmospheric Chemistry of Early Earth and terrestrial type exoplanets.}

What was the Sun's impact in tandem with geophysical factors on the chemistry of the secondary atmospheres of terrestrial planets and the initiation of prebiotic chemistry? The key question is: What observations and laboratory experiments can best help explore terrestrial planet prebiotic chemistry exposed to the Sun's ionizing irradiation? The Kepler Space Telescope, in its mission to discover exoplanets, has characterized frequent and energetic flares (or superflares) on solar-type stars, providing a mechanism by which host stars may have profound effects on the physical and chemical evolution of exoplanetary atmospheres [7,8]. The Kepler survey provided a clear correlation between maximum flare energy and stellar activities represented by a starspot area that can be extended to predict possible maximum impacts of stellar energetic particle events (SEPs) for individual exoplanets from their host stars $[8,23]$. These effects may represent a vastly underestimated factor regarding the chemical impacts of energetic particles, including SEPs and the relative contribution from Galactic Cosmic Rays. The Earth's middle atmospheric chemistry during large SEPs is represented in the production of NOx, HOx constituents, and destruction of ozone [24-26]. These studies aim to answer the following three crucial questions: (1) What are the strongest biosignatures of an Earth-like biosphere that experiences XUV/SEP fluxes? (2) How significant is ozone destruction in oxygenated exoplanetary atmospheres of Earth-like planets around G-M dwarfs? (3) What are the e ffects of superflare-induced SEPs on the prebiotic chemistry of rocky exoplanets around G-M dwarfs - and at what level can the particle radiation be lethal for surface life forms?

Life requires the formation of biologically important molecules that can create chemical disequilibrium by forming complex molecules from simpler species that ultimately produced life forms - the process is known as abiogenesis. This rise of complexity could be driven as a by-product of persistent energy sources, including UV radiation, shock heating from impacts, electric discharges, radioactivity, and solar and cosmic ray particles [1,7,27-32]. The crucial, raw atmospheric molecular ingredients of life as we know it include $\mathrm{N}_{2}, \mathrm{CO}_{2}, \mathrm{CH}_{4}$ and $\mathrm{H}_{2} \mathrm{O}$. The definition of a habitable zone has recently been enhanced to a biogenic zone (BZ) or abiogenesis zone, within which the stellar ionizing energy fluxes are efficient enough to ignite reactive chemistry through ionization and dissociation of these molecular ingredients [4,33]. Hydrogen cyanide, the major product, mixes with water vapor and forms formamide that may serve as a 
prerequisite for the complex molecules crucial for life [34,35]. This chemistry also forms $\mathrm{N}_{2} \mathrm{O}$, a powerful greenhouse gas and possible biosignature that may keep the atmosphere warm for liquid surface water to exist. Recent studies of chemical impacts of exoplanetary atmospheres around active stars show the efficient production of $\mathrm{NOx}$ and $\mathrm{OHx}$, which scale linearly with the particle fluxes [24,36,37]. Moreover, emerging laboratory experiments show that the enhanced irradiation increases the production rates of amino acids and more complex molecules, including adenine [38-40]. These studies are providing necessary context for the future detection of Earth-like biospheres' signatures around active stars.

To address this topic, the community needs to perform a comprehensive comparative planetology study of the evolution of the atmospheres, interiors, and climates of Venus, Earth, and Mars under the young Sun. Early Venus received twice the flux of the solar ionizing radiation than early Earth did. In the meantime, Mars, with a surface gravity of only $38 \%$ of the Earth's, received less than twice the solar radiation flux, but had undergone a massive atmospheric escape in its early history. We should use the geological record on Earth to constrain our models of the magma ocean and primary atmosphere, the subsequent formation of Earth's core, the onset of its magnetic dynamo, mantle convection, and the formation and co-evolution of the secondary atmosphere with atmospheric escape driven by the young Sun and apply them to early Venus and Mars. The following major questions are needed to be addressed:

1. What chemistry of the most abundant and biologically important molecules that participate in pathways producing complex sugars, amino acids, and nucleobases can be learned from the biochemistry community studying the origin of life on Earth? 2. How can we detect spectral signatures of prebiotically important molecules fundamental prerequisites of life, including nitric oxide, hydrogen cyanide, and nitrous oxide? 3. How can astrophysics inform laboratory experiments in understanding which pathways efficiently produce biologically essential molecules?

4. What steps are needed to build a unified network of theorists, observers, and laboratory scientists to explore the most efficient, laboratory validated, and calibrated methodologies to characterize the biologically important molecules with the strongest spectral signatures (high signal-to-noise, low spectral resolution) of life?

We need to design experiments to characterize production rates for the synthesis of higherorder species, including $\mathrm{HCN}, \mathrm{HCHO}, \mathrm{NH}_{3}$, and $\mathrm{NOx}$ from UV-SEP promoted synthesis. These production rates of species will then be related to their removal rates by various pathways, including aqueous reactions in aerosols, atmospheric rainout and aqueous phase dark and photochemical reactions once delivered to planetary surfaces. This will enable plausible fluxes and concentrations of these species in primitive surface environments to be constrained. These experiments will help evaluate the plausibility of various scenarios for prebiotic organic synthesis, and whether UV and SEP's can provide sufficient quantities of species thought to be required for various prebiotic chemical syntheses. Exploring how these environments co-evolved in the early Solar System and how 
universal they may be are crucial to understand the initiation of prebiotic chemistry on terrestrial planets and to develop methods to search for habitable exoplanets. We conclude that the paramount goal must be to break barriers between chemists and other laboratory scientists applying the methodologies and tools of newly formed Prebiotic Chemistry and Early Earth Environments (PCE3) and Nexus for Exoplanetary System Studies (NExSS) networks for the benefit of developing viable strategies for defining and searching the signatures of life in the Universe.

\section{References:}

[1] Airapetian, V.S. et al. (2020) Int. J. Astrobiology 19, 136; [2] Schrijver, C. J. et al. (2015) Advances in Space Res., 55, 2745; [3] Airapetian et al. NatGeo, 9, 452, 2016; [4] Airapetian et al. (2017), ApJL, 817, L24;[5]Dong et al. PNAS, 115, 260, 2018; [6] Boro Saikia et al. (2019), A\&A, 635, A178; [7]Maehara, H. et al. (2012) Nature, 485, 478; [8] Notsu, Y. et al. (2019) ApJ, 876, 58; [9] Fu, J. et al. 2019, ApJL, 878, L36; [10] Rosén, L. et al. (2016), A\&A, 593, A35; [11] Cohen, O. et al. (2004), ApJ, 790, 57; [12] Johnstone, C. et al. 2019, A\&A, 624, L10; [13] Glocer, A. et al. (2019) AGUFM, P22B-08; [14] https:/lccmc.gsfc.nasa.gov; [15] France et al. (2016), ApJ, 820,24; [16] Hinkel, N. et al. (2017), ApJ,848,19; [17] Lynch et al. (2019), ApJ, 880, 97; [18] Garcia-Sage et al. (2017), ApJL 844, L13; [19] Luger,R. and Barnes, R. (2015), Astrobiology, 15, 119; [20] Tian et al. (2014), Earth \& Plan. Sci. Let, 385, 22; [21] Luger et al. ApJ, (2017), 837,17; [22] Meadows, V. et al. (2017), Astrobiology, 17, 1022;[23] Trottet, G. et al. (2015), Sol. Phys., 290, 819; [24] Airapetian et al. (2017) Nature Sci. Rep, 7; [25] Jackman, C. et al. (2005),JGR, 100, A09S27; [26] Herbst et al. (2019), A\&A, 631, A101; [27] Miller, S.L. \& Urey, H.C. Science, 130,245, 1959; [48] Maher,K.A. \& Stevenson,D.J. Nature,331,612, 1988; [29] Chyba, C. \& Sagan,C. Nature,355,125, 1992; [30] Adam,Z. et al. Phil.Trans.Royal Soc., 375,20160348, 2017; [31] Navarro-González et al. (1998), GRL, 25,3123; [32] McKay et al. (1988), Nature, 332(6164), 520; [33] Rimmer, P. et al. (2018), Science Advances, 4,1; [34] Bada, J. et al. (2016) Phys. Chem. Chem. Phys., 18, 20085; [35]Saladino, R. et. al. (2016), OLEB, 46, 515; [36] Tabataba-Vakili et al. (2016) A\&A, 585, A96, 2016; [53] Grenfell, J. L. (2017) Phys. Rep.,713,1; [38] Kobayashi, K. et al. (1998), OLEB, 28, 155; [39] Kobayashi, K. (2019), in Astrobiology, 43; [40] Kobayashi, K. et al. (2017) XVIIIth Int. Conf. on the Origin of Life, LPI No. 1967, d.4133 [40] 